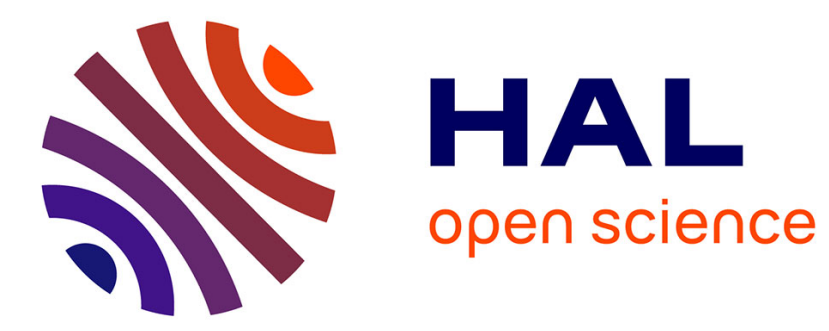

\title{
Experimental and numerical investigation of multipactor discharges in a coaxial waveguide
}

I A Kossyi, G S Luk'Yanchikov, V E Semenov, N A Zharova, D Anderson, M Lisak, J Puech

\section{- To cite this version:}

I A Kossyi, G S Luk'Yanchikov, V E Semenov, N A Zharova, D Anderson, et al.. Experimental and numerical investigation of multipactor discharges in a coaxial waveguide. Journal of Physics D: Applied Physics, 2010, 43 (34), pp.345206. 10.1088/0022-3727/43/34/345206 . hal-00597828

\section{HAL Id: hal-00597828 \\ https://hal.science/hal-00597828}

Submitted on 2 Jun 2011

HAL is a multi-disciplinary open access archive for the deposit and dissemination of scientific research documents, whether they are published or not. The documents may come from teaching and research institutions in France or abroad, or from public or private research centers.
L'archive ouverte pluridisciplinaire HAL, est destinée au dépôt et à la diffusion de documents scientifiques de niveau recherche, publiés ou non, émanant des établissements d'enseignement et de recherche français ou étrangers, des laboratoires publics ou privés. 


\title{
Experimental and Numerical Investigation of Multipactor Discharges in a Coaxial Waveguide
}

\author{
I.A. Kossyi, G.S. Luk'yanchikov \\ Prokhorov Institute of General Physics, Russian Academy of Sciences, \\ 38 Vavilova Street, Moscow, 119991 Russia \\ V.E. Semenov, N.A. Zharova \\ Institute of Applied Physics, Russian Academy of Sciences, \\ 46 Ulianov St., Nizhny Novgorod 603950, Russia \\ D. Anderson*, M. Lisak \\ Department of Radio and Space Science, \\ Chalmers University of Technology, 41296 Goteborg, Sweden \\ ${ }^{*}$ Corresponding author: elfda@chalmers.se \\ J. Puech \\ Centre National d'Etudes Spatiales, 31401 Toulouse, France
}

\begin{abstract}
An experimental and numerical investigation is made of multipactor discharges in a coaxial waveguide. Particular attention is given to a determination of the multipactor threshold and the distribution of the impact energy of the electrons. Simulations are carried out for different parameters of the secondary emission coefficient of the electrode surfaces. This makes it possible to determine these parameters through a comparison between the numerical and experimental results. The comparison also shows that the observed multipactor is mainly of polyphase (non-resonant) nature and represents a mixture of single- and double-surface multipactor discharges.

PACS numbers: 52.80.Pi, 52.80.Vp
\end{abstract}




\section{INTRODUCTION}

Multipactor discharges constitute a potentially severe problem for modern microwave systems involving high powers and operating close to vacuum conditions [1-7]. The multipactor phenomenon has been studied intensively both experimentally, theoretically, and numerically during more than 50 years [8-18]. However, comparisons between experimental and theoretical results present a difficult problem. On one hand, multipactor experiments do not in general involve detailed measurements of the secondary emission properties of the solid surfaces which generate the multipactor electron avalanche. On the other hand, numerical results are usually obtained using some particular model for the secondary electron emission yield and the sensitivity of the concomitant results on this model is not clear. Some data on the secondary emission properties for different materials can be found in the ESA standard [19]. However, these data do not take into account the contamination of solid surfaces that is present in realistic experiments. The secondary emission properties are known to be very sensitive to different surface contaminations and consequently the simulations which are carried out using standard emission data may deviate significantly from the experimental results [20]. However, the problem can be turned around in such a way that a comparison between numerical calculations and measurements is instead used to obtain information concerning the (unknown) parameters of the secondary emission yield.

A determination of the multipactor threshold is the most simple and common measurement in an experiment. In order to obtain a comprehensive comparison of these measurements with theory, it is necessary to know the dependence of the multipactor threshold on frequency and the geometrical parameters of the system [2, 21-24]. However, having access to additional experimental data (i.e. not only the multipactor threshold) and carrying out numerical simulations within a wide range of parameters, it is possible to determine the parameters of the secondary emission yield even in the case of fixed system geometry and frequency. For example, in [20] such additional information was obtained by experimental investigation of multipactor discharges in a rectangular waveguide within a wide range of transmitted microwave powers and using independent measurements of the first cross-over energy of the secondary emission yield in the waveguide. In the present paper, multipactor breakdown in a coaxial transmis- 
sion line is investigated experimentally and simulated numerically. The geometry of the coaxial line, as well as the microwave frequency, was kept fixed. In contrast to [20] it was not possible to measure the first cross-over energy in the waveguide. Instead the distribution of the impact energy of the multipacting electrons was measured in the experiment, as suggested in [24]. This information made it possible to do a comprehensive comparison with numerical simulations and was also used to confirm the poly-phase nature of the multipactor discharge [25-29].

\section{EXPERIMENTAL SETUP}

A schematic picture of the experimental setup used for investigating multipactor discharges in a coaxial cylindrical waveguide is shown in Fig. 1. The central section of the coaxial waveguide 1 with an outer electrode (made of brass) of radius $R_{\text {out }}=12.5$ $\mathrm{mm}$ and an inner electrode (made of duralumin) of radius $R_{i n}=3 \mathrm{~mm}$ is placed in the vacuum chamber 3 . Within the chamber, the waveguide narrows linearly to the radii of $R_{\text {out }}=4 \mathrm{~mm}$ and $R_{\text {in }}=2 \mathrm{~mm}$. The outer electrode has a set of holes for pumping out the waveguide and (in the case of a multipactor discharge) for extracting the electron current to the ring collectors $K_{1} \div K_{8}$. The diameter of the holes was small enough $(0.5 \mathrm{~mm})$ to avoid significant perturbations of the electromagnetic field in the waveguide. Four collectors $K_{1} \div K_{4}$ are arranged along the narrowest cylindrical part of the waveguide (of length $L=5 \mathrm{~cm}$ ), whereas the other $K_{5} \div K_{8}$ collectors are installed in its expanding conical part (more specifically in the cross-sections where the radius of the outer electrode equals $4.4,4.7,5.8$, and $10.8 \mathrm{~mm}$ respectively). The electric current from the collectors was recorded using an oscilloscope (Tektronix TDS 220), which visualized the time evolution of the current. The potential bias between the collector and the outer electrode could be varied and the dependence of the collector current on this potential bias could be determined. Thus, information concerning the energy distribution of the impacting electrons [24] could be obtained. This made it possible to measure also the ionic current (if any) to the collectors and thereby to distinguish the electron multipactor effect from the plasma effect caused by micro-sparks [20, 27].

The fluoro-plastic windows 2 separated the central vacuum section of the waveguide 


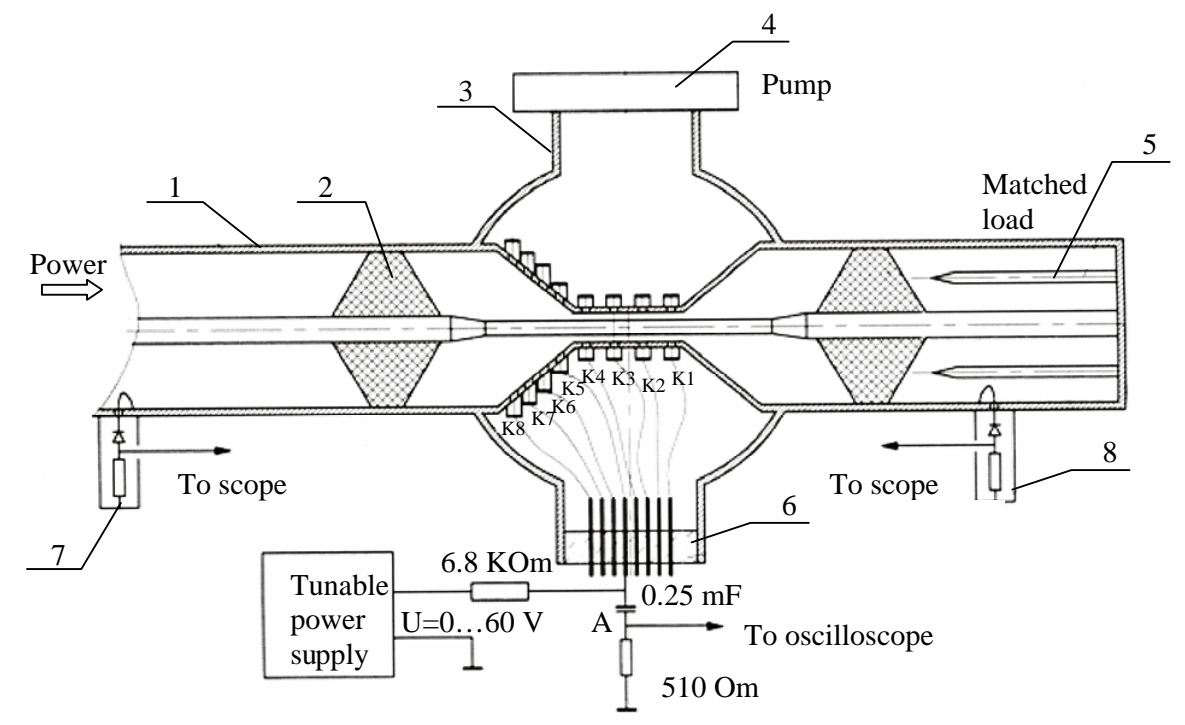

FIG. 1: Scheme of the experimental setup: (1) coaxial waveguide, (2) fluoro-plastic windows, (3) vacuum chamber, (4) electric-discharge vacuum pump, (5) matched load, (6) multi-pin input for power supply of the collectors and $(7,8)$ microwave detectors. Collectors $K_{1} \div K_{8}$ record the electron/ion current depending on the dc bias applied.

in the chamber from the side sections, which were filled by air at atmospheric pressure. The right (edge) waveguide section was held at atmospheric pressure and equipped with the matched load 5 to realize the regime of a travelling electromagnetic wave in all sections of the coaxial line and to keep the power reflection coefficient very low (less than $0.25 \%$ ). To reduce the possibility of the appearance of micro-sparks at the metal-dielectric interface [30,31], the windows were positioned in the widest portion of the coaxial waveguide, where the microwave electric field is weakest. Furthermore, to reduce the density of the plasma that can result from the micro-sparks, the joint between the window and the inner electrode was sealed by a narrow circular groove. The chamber and waveguide are pumped out to a pressure of $2 \div 3 \cdot 10^{-6}$ Torr by the oil-free electric-discharge (titanium) pump 4. The fundamental mode in the coaxial line was excited at the frequency $f=2.45 \mathrm{GHz}$ by a microwave generator (magnetron) that operated in the pulse repetition regime with pulse duration $\tau_{f}=1 \mathrm{~ms}$, peak power $P \leq 3 \mathrm{~kW}$ and repetition frequency $F=0.4 \mathrm{~Hz}$. The detector heads 7 and 8 measure the microwave field intensity in the input and output sections of the waveguide. The 
field intensity in these sections depends on the wave reflection and the absorption that would be caused by a discharge in the waveguide. Therefore it was possible to identify such a discharge using signals from detectors 7 and 8 .

\section{EXPERIMENTAL RESULTS}

Figures 2 and 3 present typical oscillograms of the signal obtained from the microwave detectors 7 and 8 in the qualitatively different cases of microwave powers below and above the breakdown threshold. At low microwave power ( $P \leq 0.9 \mathrm{~kW})$, the signals from both detectors have a similar profile - a replica of the profile of the microwave pulse (see Fig. 2). However at higher power levels ( $P \geq 1 \mathrm{~kW}$ ), the signal profiles are qualitatively different (Fig. 3) and demonstrate an abrupt jump (up or down) in the field intensity after a temporal delay that depends on the excess power above the threshold. In the input section, a sharp increase in the microwave field intensity is observed which can be understood as a result of constructive interference between the incident electromagnetic wave and the reflected electromagnetic wave that appears due to the presence of the discharge in the waveguide. At the same time the field intensity in the output section decreases as a result of both partial reflection and absorption of the incident wave.

The signals from collectors $K_{1} \div K_{8}$ present additional information on the discharge properties. At low microwave powers, the electric current from all collectors is zero during the pulse whereas at higher power levels current pulses are recorded from all collectors, although with slightly different time delays and different peak values (Fig. 3). The current profile is found to be qualitatively similar for all collectors. Typically it starts with a very short peak (with a duration of about 2-10 $\mu$ s) and is followed by a considerably slower increase in the current and the establishing of a certain quasistationary level. The first peak is associated with the electron multipactor discharge since no ionic current was detected during this time. The multipactor peaks were found to appear almost simultaneously at all collectors although the values are quite different (the highest peak amplitude was detected at the collector $K_{5}$, as can be seen from Fig. 


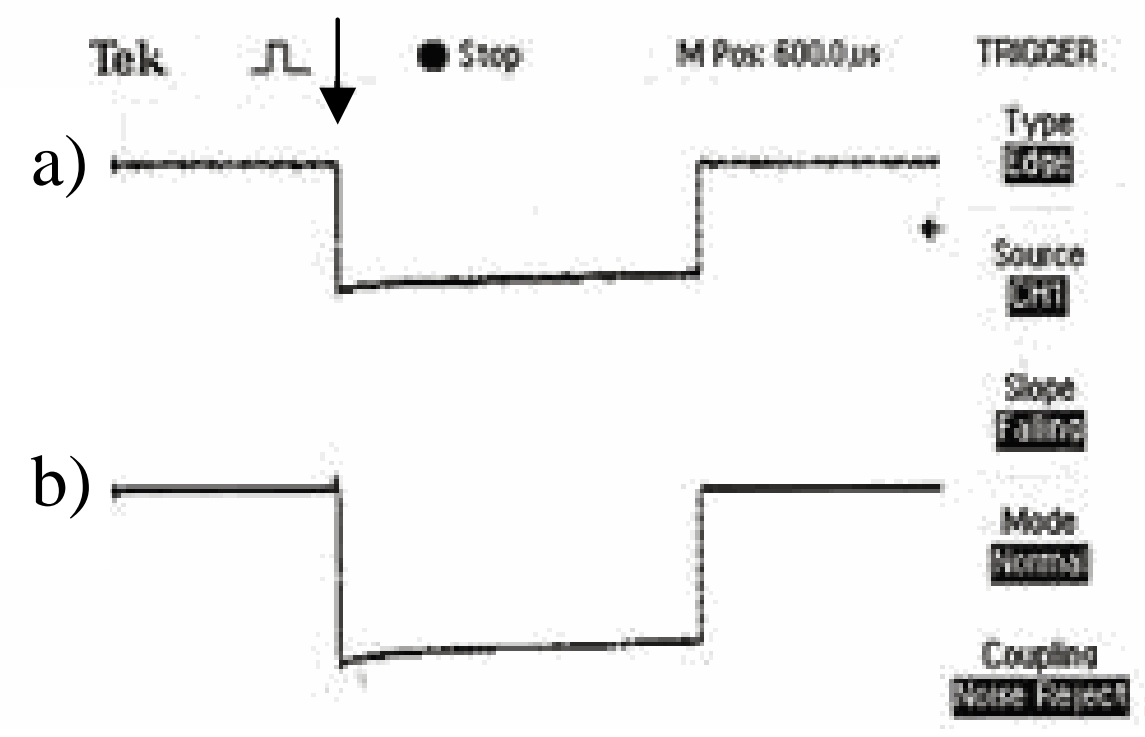

FIG. 2: Representative pulse oscillograms from the detectors at the input (a) and output (b) sections of the vacuum chamber in a case of low input power $(\mathrm{P}=0.9 \mathrm{~kW})$ when there is no discharge in the coaxial waveguide. The vertical arrow $\downarrow$ in the top line indicates the beginning of the microwave pulse.

$4)^{1}$. On the other hand, the delay of the second current peak is quite different for the different collectors. These peaks and the quasi-stationary current are associated with plasma formation due to ionization of gas desorption from the waveguide walls. An ionic current is detected at this stage when the negative potential bias between the collector and the outer coaxial electrode becomes strong enough to reflect all electrons. The electric current to the collector (if any) is then completely determined by ions. It should also be noted that perturbations of the signals from the microwave detectors (Figs. 2 and 3) are observed only after the plasma formation.

Fig. 5 illustrates the dependence of the multipactor peak current on the bias voltage applied between the collector $K_{4}$ and the outer electrode of the coaxial waveguide.

${ }^{1}$ The observed phenomena (the simultaneous formation of the first current peak at different collectors as well as the separation of the maximum current from the dielectric seal) confirm that the physical mechanism behind is not plasma jets generated by micro-sparks at the metal-dielectric interface, but rather due to multipactor discharges. 


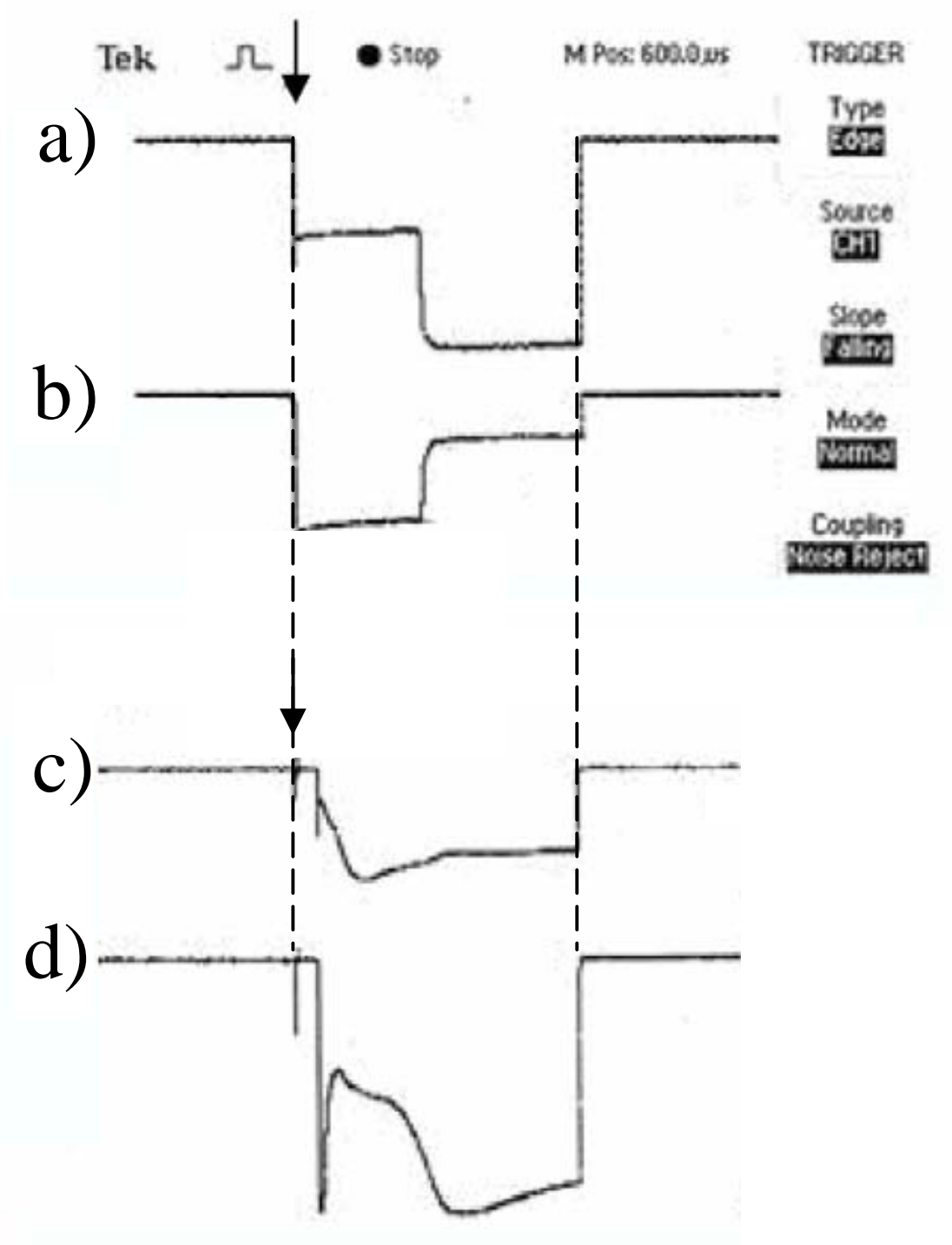

FIG. 3: The same as in Fig. 2 but in a case of high input power $(P=1.5 \mathrm{~kW})$ when there is a discharge in the coaxial waveguide. Lower part of figure shows representative oscillograms of the electron current signals from collectors $K_{8}(\mathrm{c})$ and $K_{5}(\mathrm{~d})$. The vertical arrow $\downarrow$ in the top line indicates the beginning of the microwave pulse.

The current-voltage characteristics makes it possible to determine the distribution of the electron impact energy, $W$, at the outer electrode (Fig. 6). Note that $F(W) \propto$ $d j_{e k} / d U_{p}$, where the impact energy is related to the potential as $W=-e U_{p}$. This distribution is found to be considerably different from that corresponding to a resonance multipactor discharge. In fact, resonance theory predicts a narrow peak in electron 


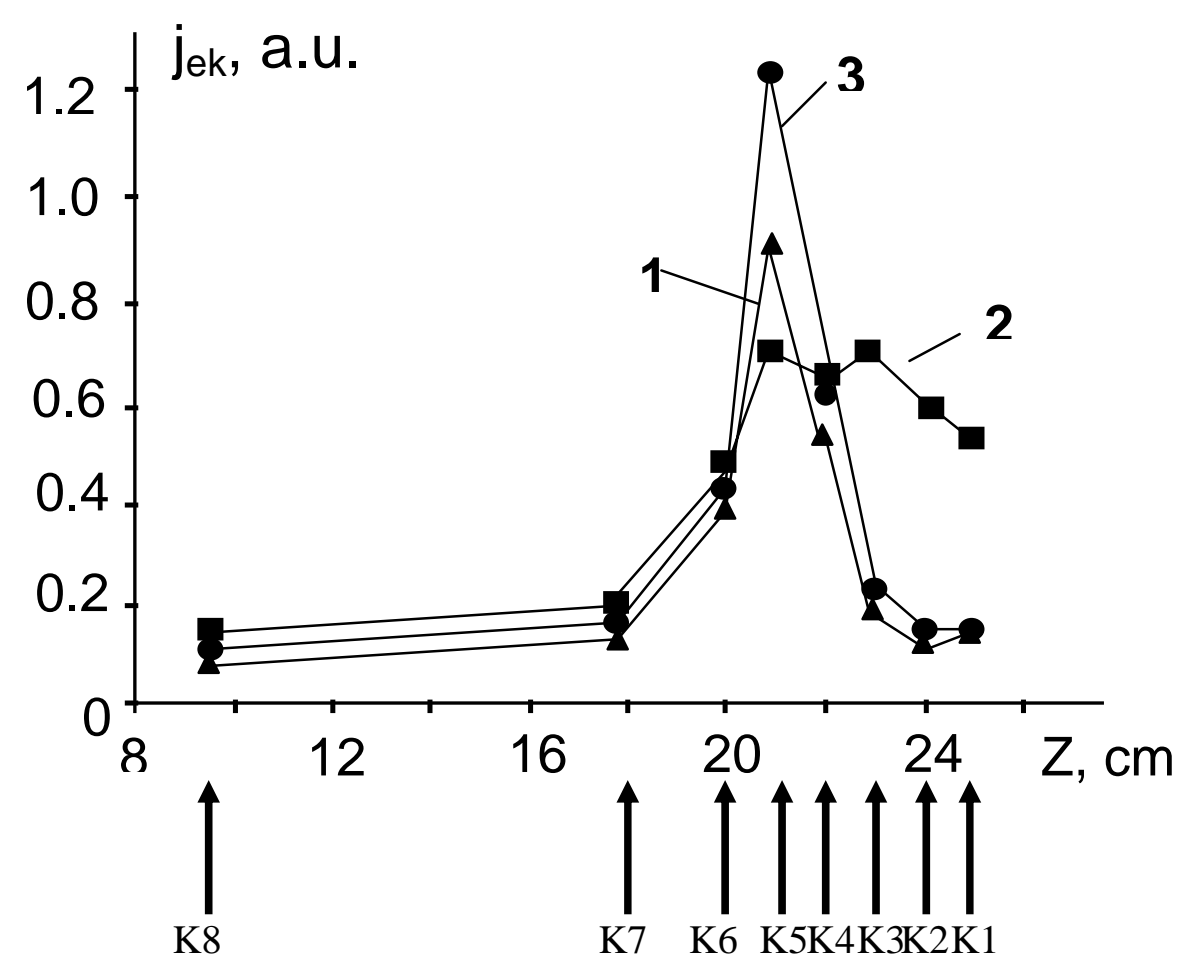

FIG. 4: Peak value (arbitrary units) of the multipactor electron current for different microwave powers and positions of the collectors along the coaxial waveguide. Each indicated point corresponds to a particular collector from $K_{8}$ (the most left) up to $K_{1}$ (the most right). Curve 1 (triangles) corresponds to $P=1 \mathrm{~kW}$; curve 2 (squares) to $P=1.5 \mathrm{~kW}$ and curve 3 (circles) to $P=3 \mathrm{~kW}$.

impact energy [32], whereas the measurements demonstrated a relatively wide spread of electron impact energy extending up to $60 \mathrm{eV}$. Fig. 6 also shows the existence of a considerable fraction of electrons with small energy (less than $10 \mathrm{eV}$ ) that can only be due to electrons returning back to the surface of emission without having experienced any significant acceleration by the microwave field.

\section{NUMERICAL SIMULATIONS}

Numerical simulations of the multipactor discharge in the coaxial line were carried out using the software COAXMUL which represents an upgrade of a previous version described in [33]. The software is based on a PIC algorithm and considers the mo- 


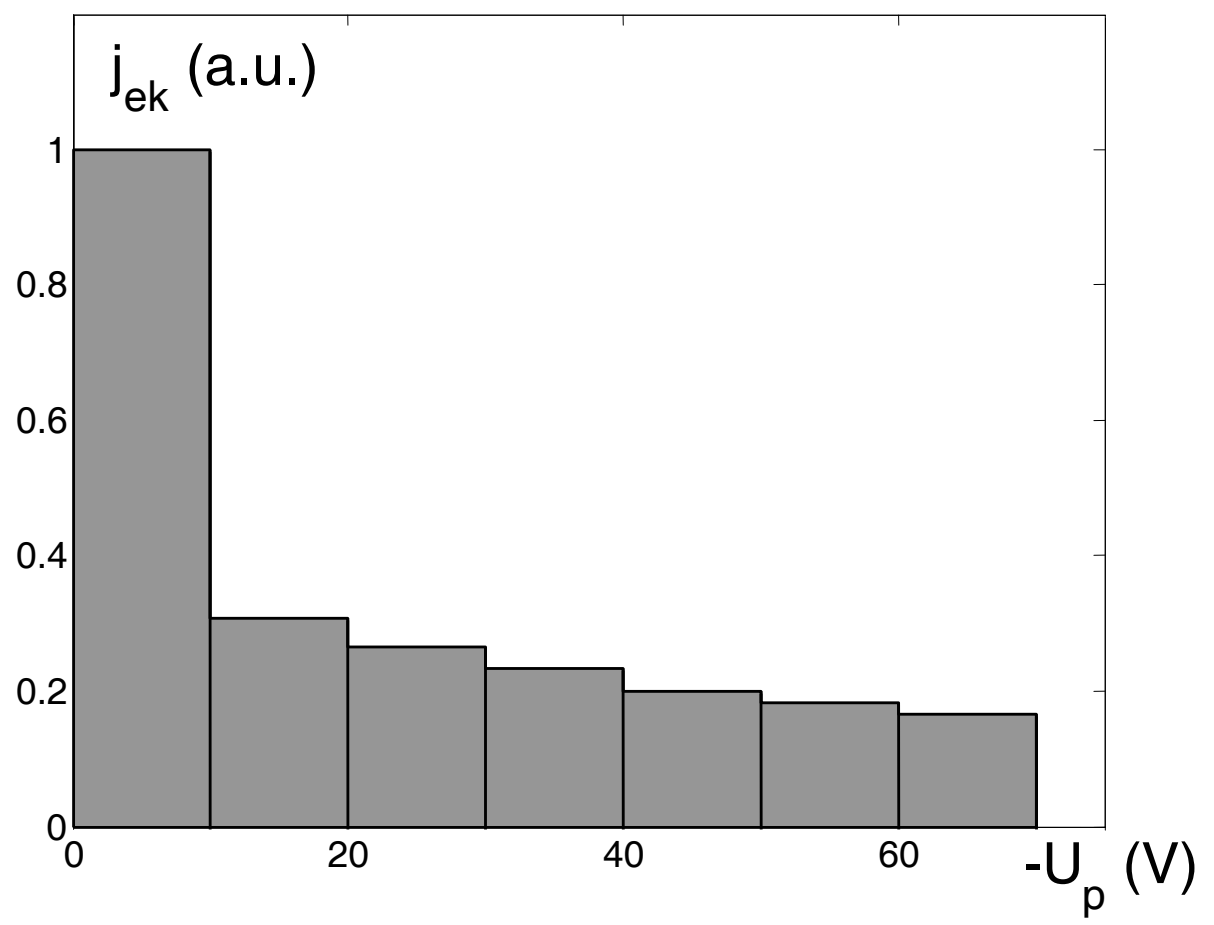

FIG. 5: Electron current to collector $K_{4}$ (arbitrary units) vs. electron decelerating potential $-U_{p}$ for $P=1.5 \mathrm{~kW}$.

tion of macro-particles (subsequently named computer particles) which have the same charge to mass ratio as the electrons. The simulations are started by the launching of seed particles having stochastic initial velocities governed by a Maxwellian probability distribution. These particles move under the action of the electromagnetic field and release a number of secondary particles when they collide with the metal walls. The secondary emission is considered as a stochastic process with a probability distribution governed by the impact energy of the particles and chosen so as to correspond to Vaughan's approximation [34] for the average value of the secondary emission yield. The secondary particles are assumed to start with stochastic initial velocities governed by the same Maxwellian probability distribution as the seed particles.

In conventional PIC softwares, the charge and mass of the computer particles are fixed during the simulations whereas the number of the particles varies. It should be emphasized that using a small number of computer particles leads to considerable stochastic fluctuations in the results whereas a large number of computer particles requires long simulation times. Therefore it is difficult to simulate the long time evolu- 


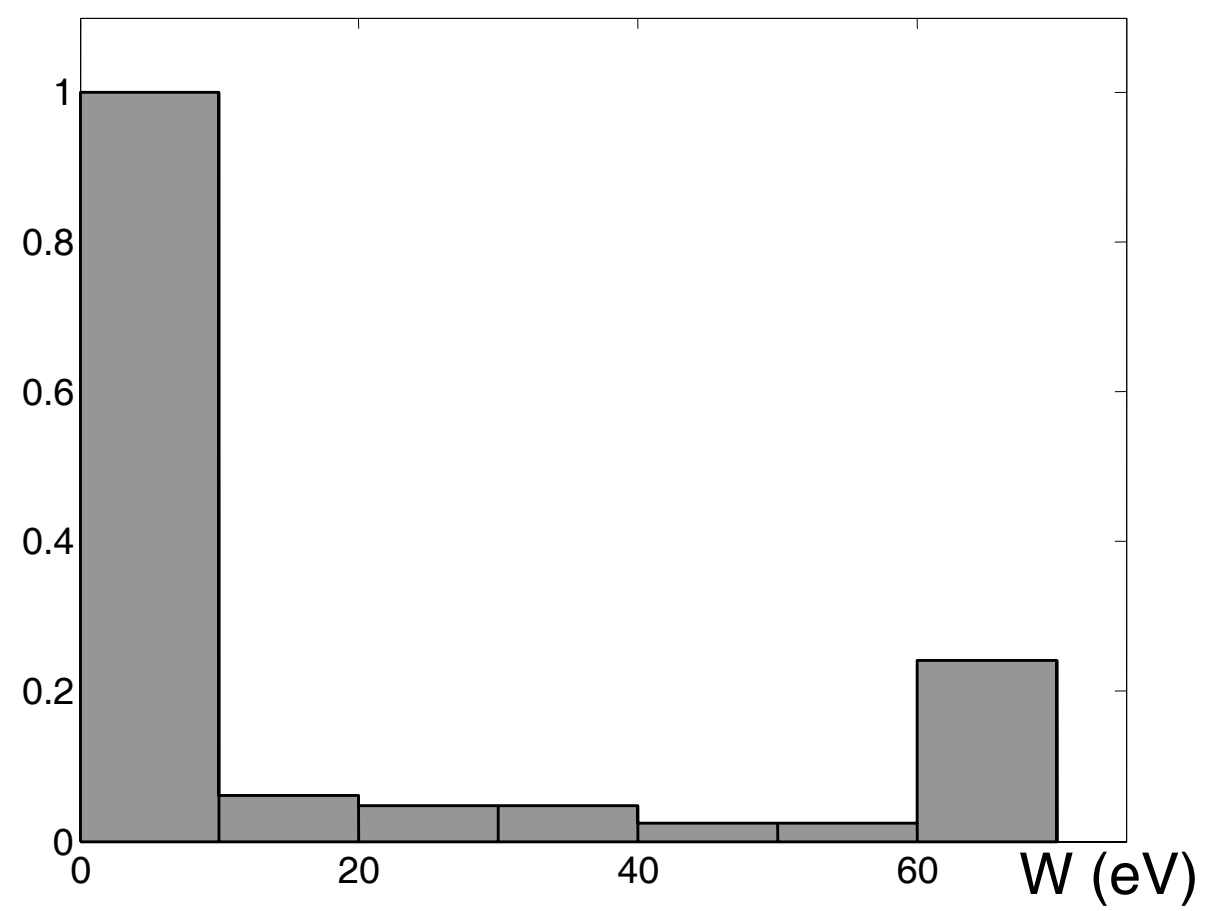

FIG. 6: Distribution of electron impact energy obtained from the current-voltage characteristic shown in Fig. 5.

tion of the multipactor. To avoid this problem in the software COAXMUL, the charge and mass of the computer particles are not considered as fixed (only the ratio between these quantities is kept fixed). This makes it possible to keep the number of computer particles within a desirable range by using the following prescription: When during the course of the simulation, the number of computer particles exceeds some threshold value, $N_{t h}$, the software excludes randomly one-half of these particles from further consideration and simultaneously doubles the charge and mass of the remaining particles. On the other hand, if the number of computer particles becomes less than $0.3 N_{t h}$, the software splits each computer particle into two new particles having one-half of the previous charge and mass. This procedure makes it possible to study the long time evolution of the multipactor avalanche while still having high simulation speed and accuracy.

It is important to note that the software uses the macro-particle mass and charge only to calculate the total electron number which is determined as the ratio of the total mass of all macro-particles to the mass of the single electron. The trajectory of 
each macro-particle is determined by its charge-to-mass ratio and consequently this trajectory and also the impact velocity coincide completely with the trajectory and the impact velocity of a single electron. This means that any macro-particle can be treated as the corresponding number of electrons having the same coordinate and velocity. A collision of a macro-particle with a solid surface is treated as a collision of the corresponding number of electrons with this surface. This process is accompanied by a release of secondary electrons, the number of which is determined by the electron impact energy or by the impact velocity of the macro-particle. However, within the software the secondary electrons are again integrated into the macro-particles having the same mass and charge as the impacting macro-particle.

The first series of simulations was carried out to study the dependence of the initial stage of the multipactor avalanche on the secondary emission properties of the waveguide walls and the microwave power transmitted through the waveguide. The number of seed particles (having the conventional electron charge and mass) was taken to be $N_{0}=25000$ and these particles were launched from the surface of the inner electrode during the first microwave period. The inner and outer electrode radii were taken to be $2 \mathrm{~mm}$ and $4 \mathrm{~mm}$ respectively, the microwave frequency was $2.45 \mathrm{GHz}$ and the threshold value of the computer particles was $N_{t h}=50000$. In these simulations, the number of electrons, $N$, was recorded after 100 microwave periods and the relative increase in the electron number, $N / N_{0}$, was plotted against the microwave power, $P$, for different combinations of such parameters as: average initial energy of electrons, $W_{s}$, first cross-over point, $W_{1}$, and maximum value, $\sigma_{\max }$, of the secondary emission curve. The results (Figs. 7 and 8) demonstrate that the threshold power for the multipactor avalanche (which corresponds to the equality $N / N_{0}=1$ ) is sensitive to the values of $W_{s}$ and $W_{1}$ whereas its dependence on the value $\sigma_{\max }$ is less pronounced. On the other hand, Fig. 9 clearly illustrates that the threshold power $900 \mathrm{~W}$ (the same as that observed in experiments) can be realized using considerably different combinations of parameters (for instance, $W_{s}=1 \mathrm{eV}$ and $W_{1}=10 \mathrm{eV}$ or $W_{s}=5 \mathrm{eV}$ and $W_{1}=20$ $\mathrm{eV})$. The multipactor simulations were repeated for these parameter combinations, but using a larger value of $N_{t h}\left(N_{t h}=2 \cdot 10^{5}\right)$ and studying the temporal evolution of the electron number, the power deposition on the electrode surface and the impact elec- 


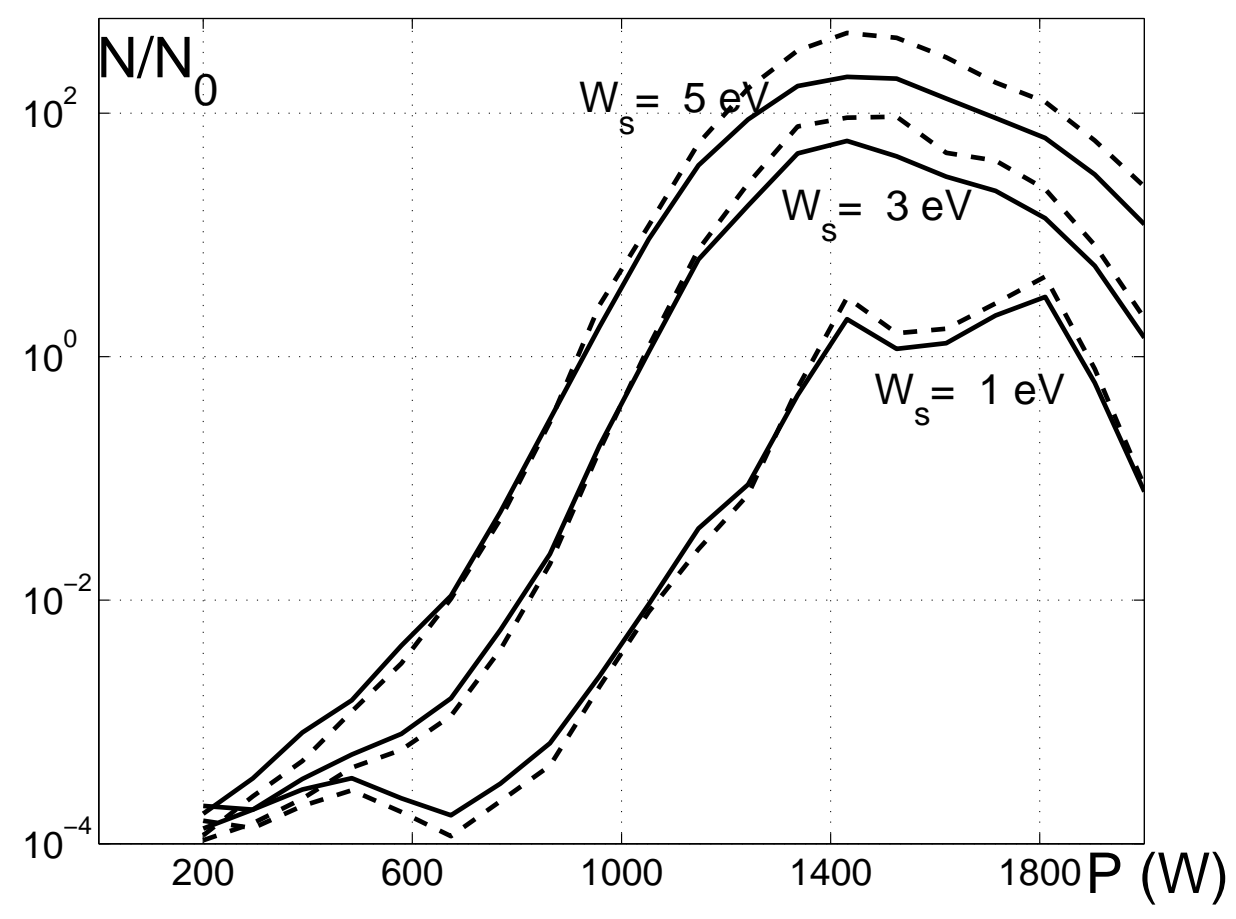

FIG. 7: The dependence of the relative increase of the electron number, $N / N_{0}$, after 100 microwave periods on the microwave power, $P$, and the properties of the secondary emission yield of the waveguide walls. All simulations were completed taking the same coaxial waveguide with electrode radii $R_{\text {in }}=2 \mathrm{~mm}, R_{\text {out }}=4 \mathrm{~mm}$, the same frequency $2.45 \mathrm{GHz}$ and the same value of the first cross-over energy $W_{1}=20 \mathrm{eV}$. Each curve represents results calculated for fixed maximum value of the secondary emission yield (the solid lines correspond to $\sigma_{\max }=2$, whereas the dotted lines correspond to $\sigma_{\max }=3$ ) and fixed value of the average initial energy, $W_{s}$, of emitted electrons (the chosen value is shown close to the lines).

tron energy. The microwave power was taken to be $1.5 \mathrm{~kW}$ in these simulations which were aimed at determining the distribution of the electron impact energy. At this high microwave power, the growth of the multipactor avalanche was faster in the case of $W_{s}=1 \mathrm{eV}$ and $W_{1}=10 \mathrm{eV}$ (Fig. 9). The evolution of the impact electron energy shows a sequence of peaks, shifted with respect to each other at the inner and outer electrodes. The amplitudes of the peaks in impact energy are similar in both cases (Fig. 10). However, one can clearly see that in the first case $\left(W_{s}=1 \mathrm{eV}\right.$ and $W_{1}=10$ $\mathrm{eV}$ ), the duration of the impact energy peaks is shorter and their amplitude is higher at the outer electrode. The difference between the two cases becomes more pronounced 


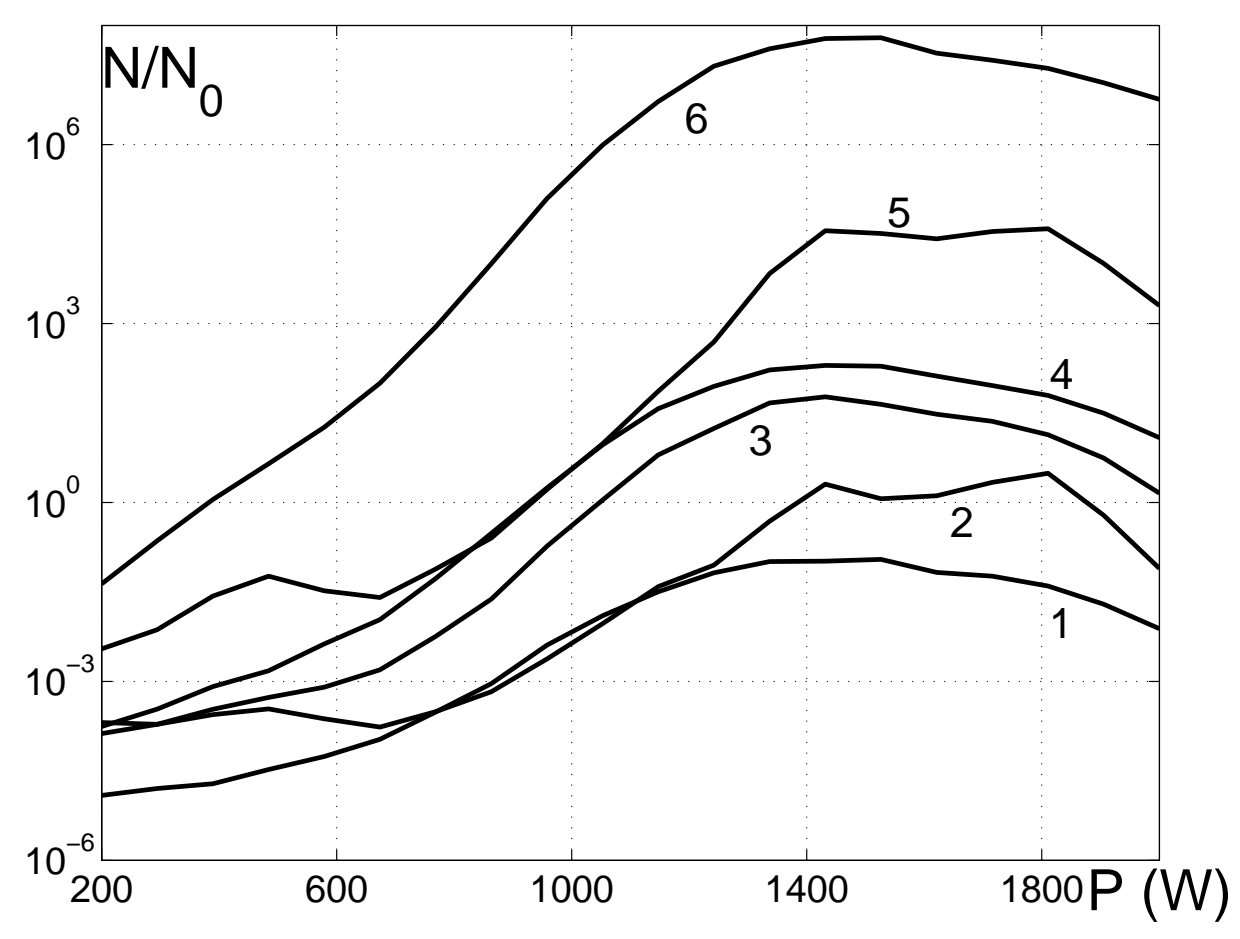

FIG. 8: The same as in Fig. 7, but taking $\sigma_{\max }=2$ for all curves and different combinations of $W_{1}$ and $W_{s}$. Line 1 corresponds to $W_{1}=30 \mathrm{eV}$ and $W_{s}=5 \mathrm{eV}$; line 2 to $W_{1}=20 \mathrm{eV}$ and $W_{s}=1 \mathrm{eV}$; line 3 to $W_{1}=20 \mathrm{eV}$ and $W_{s}=3 \mathrm{eV}$; line 4 to $W_{1}=20 \mathrm{eV}$ and $W_{s}=5 \mathrm{eV}$; line 5 to $W_{1}=10 \mathrm{eV}$ and $W_{s}=1 \mathrm{eV}$; line 6 to $W_{1}=10 \mathrm{eV}$ and $W_{s}=5 \mathrm{eV}$.

when the time evolution of the heating power at both electrodes is studied (Fig. 11). In the simulations the relative heating power was calculated as follows. During each temporal interval equal to 0.05 of the microwave period, all impact energies at each electrode were summarized: $W_{\sum}=\sum W_{i}$. During the same temporal interval all initial energies of the secondary electrons were also summarized: $W_{0 \sum}=\sum W_{0 i}$. The relative heating power (during the considered temporal interval), $Q$, was then calculated as $Q=20\left(W_{\sum}-W_{0 \sum}\right) /(N T)$, where $N$ is the number of electrons in the middle of the considered temporal interval and $T$ denotes the microwave period. Specifically, in the first case electrons collide with the outer electrode more often and thereby the heating power is considerably higher there than at the inner electrode. The same asymmetry between the outer and inner electrode is observed also in the second case, but the difference in the heating power is not so pronounced in this case. Detailed simulations were also used to calculate the distribution of electron impact energy at the outer electrode 


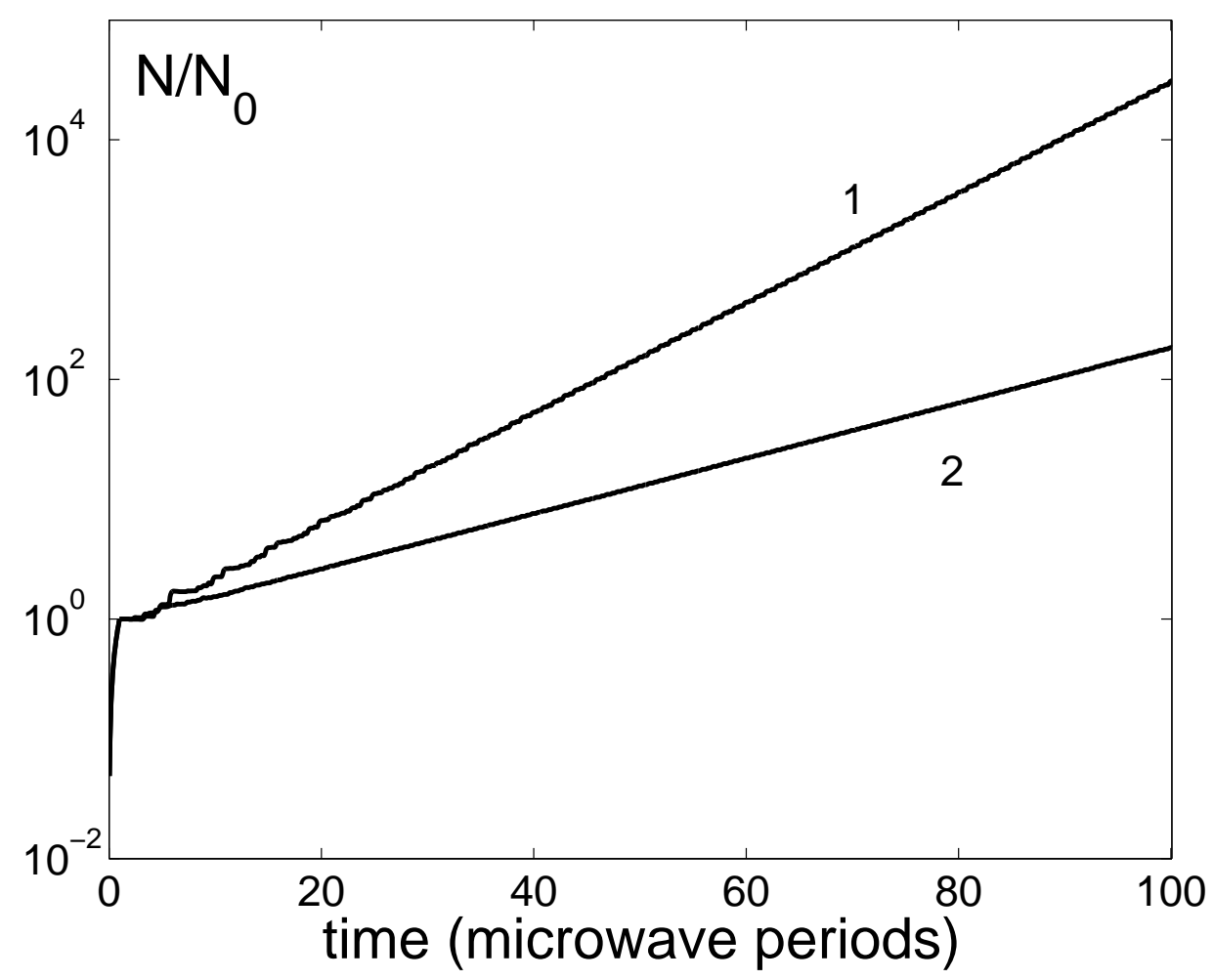

FIG. 9: The evolution of the relative number of electrons at high microwave power, $P=1.5$ $\mathrm{kW}$ and for different parameters of the secondary electron emission yield. Line 1 corresponds to $\sigma_{\max }=2, W_{1}=10 \mathrm{eV}$ and $W_{s}=1 \mathrm{eV}$ and line 2 to $\sigma_{\max }=2, W_{1}=20 \mathrm{eV}$ and $W_{s}=5 \mathrm{eV}$.

(Fig. 12). In both cases considered, the numerical results demonstrate the presence of a considerable fraction of electrons with low impact energy, in agreement with the measurements (cf. Fig. 6). Also in both cases, no electron impact energies greater than $80 \mathrm{eV}$ were found. However, in contrast to experiment, the fraction of electrons with impact energies between 20 and $60 \mathrm{eV}$ was found to be very small in the first case whereas in the second case this fraction was found to be in reasonable agreement with the measurements.

\section{DISCUSSION}

In general, the multipactor phenomenon is discussed in terms of resonance between the oscillations of the electrons and the rf electric field in the device volume [8, 11, 32]. When resonance theory is used to analyze multipactor breakdown in a coaxial line [23, 

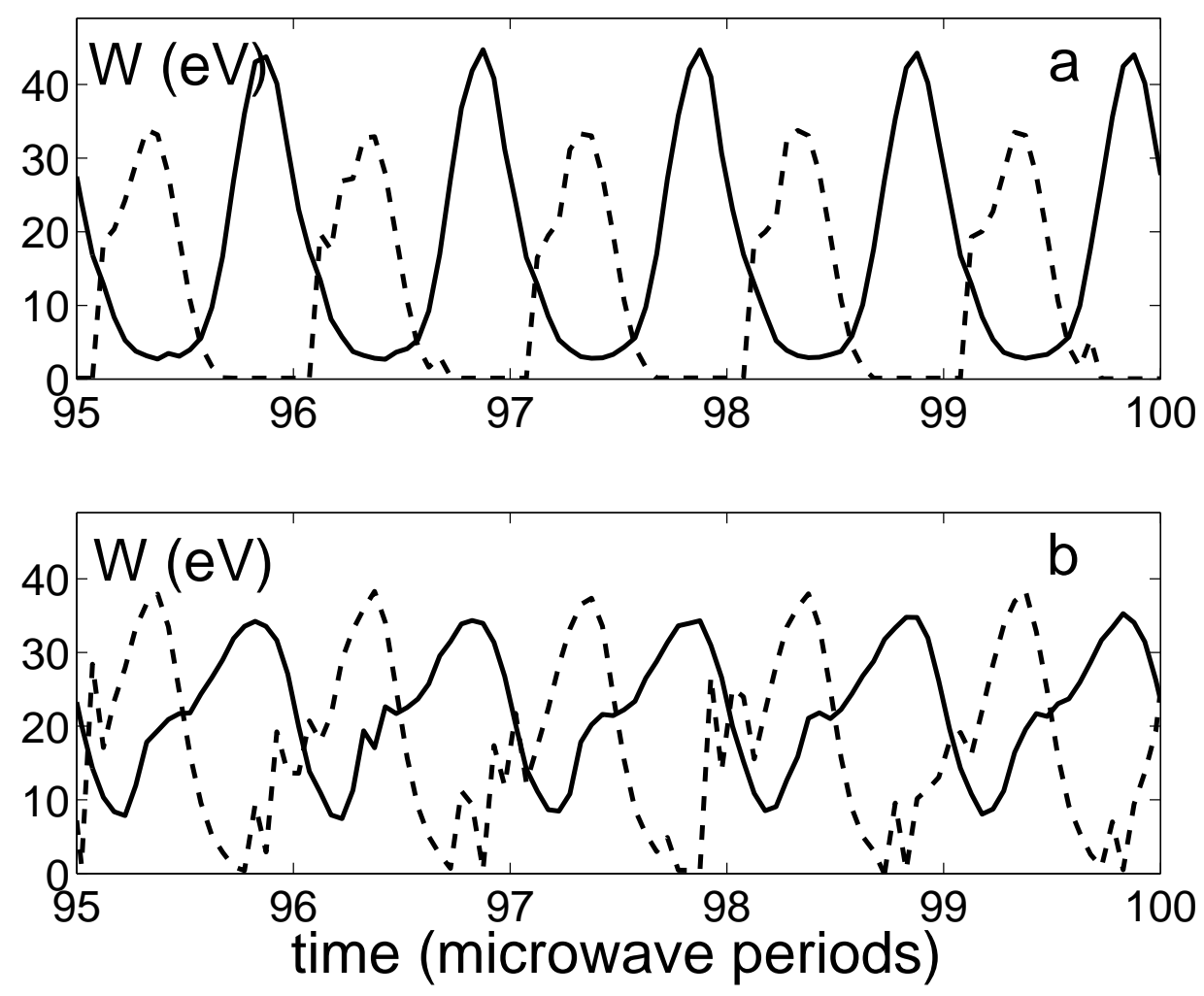

FIG. 10: The evolution of the impact electron energy, $W$, (averaged over a time interval equal to 0.05 microwave periods) at high microwave power, $P=1.5 \mathrm{~kW}$ and for different parameters of the secondary electron emission yield (Fig. 10a corresponds to $\sigma_{\max }=2, W_{1}=10 \mathrm{eV}$ and $W_{s}=1 \mathrm{eV}$ and Fig. $10 \mathrm{~b}$ to $\sigma_{\max }=2, W_{1}=20 \mathrm{eV}$ and $\left.W_{s}=5 \mathrm{eV}\right)$. The solid line represents the electron impact energy at the outer electrode, whereas the dotted line represents the impact energy at the inner electrode.

$35]$, it predicts growth of an electron avalanche only within relatively narrow separated bands of transmitted power. However, these predictions are not in agreement with our experiments where the multipactor was observed within a wide range of powers in agreement with the predictions of the poly-phase multipactor theory [25-29]. One important reason for this discrepancy is the spread of initial electron emission velocity, which is not negligible compared to the impact velocity corresponding to the first crossover point of the secondary emission curve. This statement is confirmed by the results of the numerical simulations. Actually, Fig. 7 demonstrates that separated narrow peaks (not very well pronounced) in the dependence of the relative number of electrons 

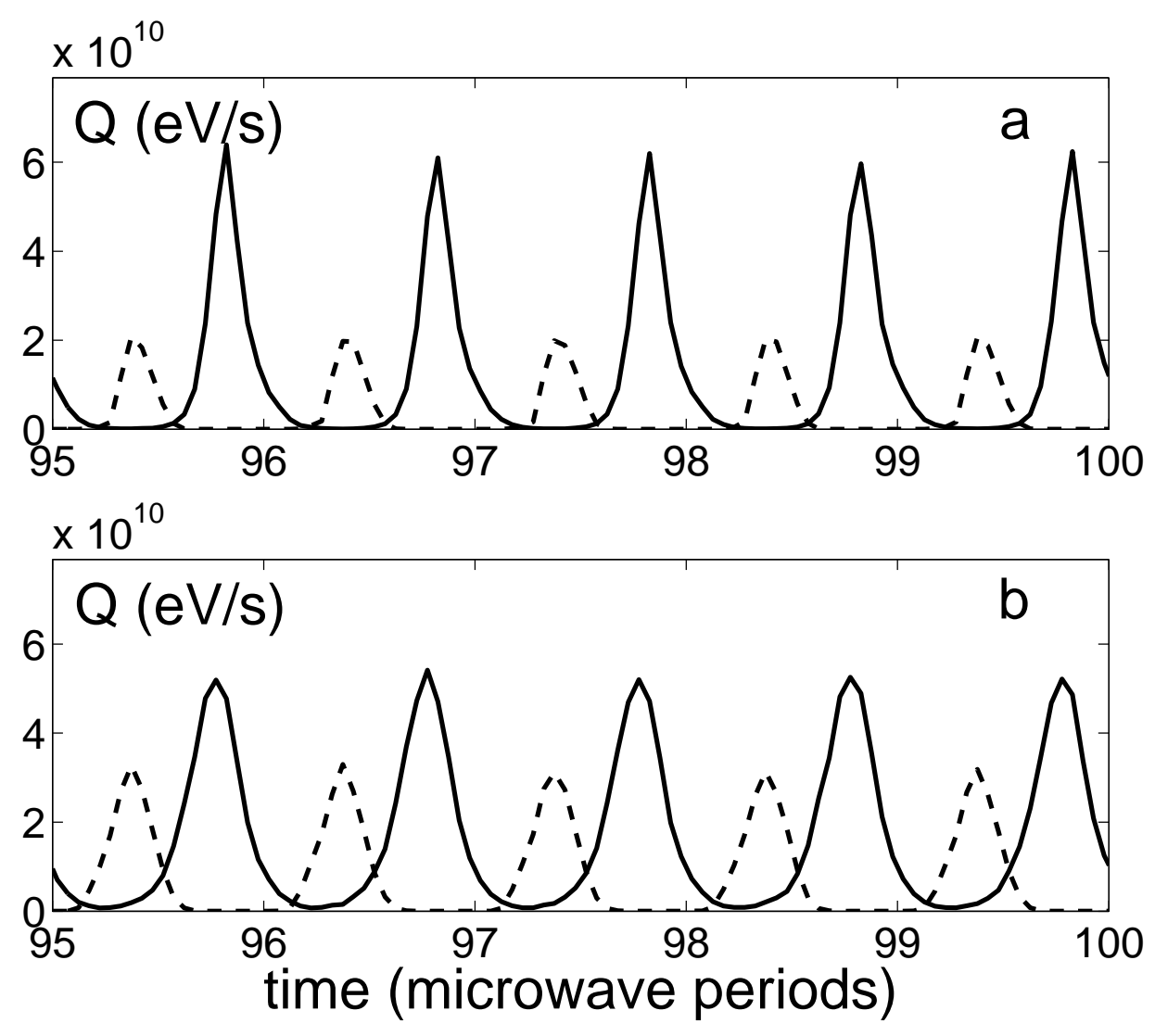

FIG. 11: The evolution of the heating power, $Q$, per one multipacting electron (averaged over a time interval equal to 0.05 microwave periods) at high microwave power, $P=1.5 \mathrm{~kW}$ and for different parameters of the secondary electron emission yield (Fig. 12a corresponds to $\sigma_{\max }=2, W_{1}=11 \mathrm{eV}$ and $W_{s}=1 \mathrm{eV}$ and Fig. $12 \mathrm{~b}$ to $\sigma_{\max }=2, W_{1}=20 \mathrm{eV}$ and $\left.W_{s}=5 \mathrm{eV}\right)$. The solid line represents the heating power at the outer electrode, whereas the dotted line represents the heating power at the inner electrode.

on microwave power appear only in the case of small initial energy, $W_{s}$, of the secondary electrons (Note that $W_{s}$ is directly proportional to the square of the electron velocity spread in the case of a Maxwellian probability distribution). Another argument for the poly-phase nature of the observed multipactor process is the wide distribution of electron impact velocities found both in measurements and in simulations (Figs. 6 and 12). It should be emphasized that also the strong periodic pulsations in electron impact energy and heating power (Figs. 10 and 11) are in agreement with the polyphase theory [27], which predicts that the probability of electron collisions with the 


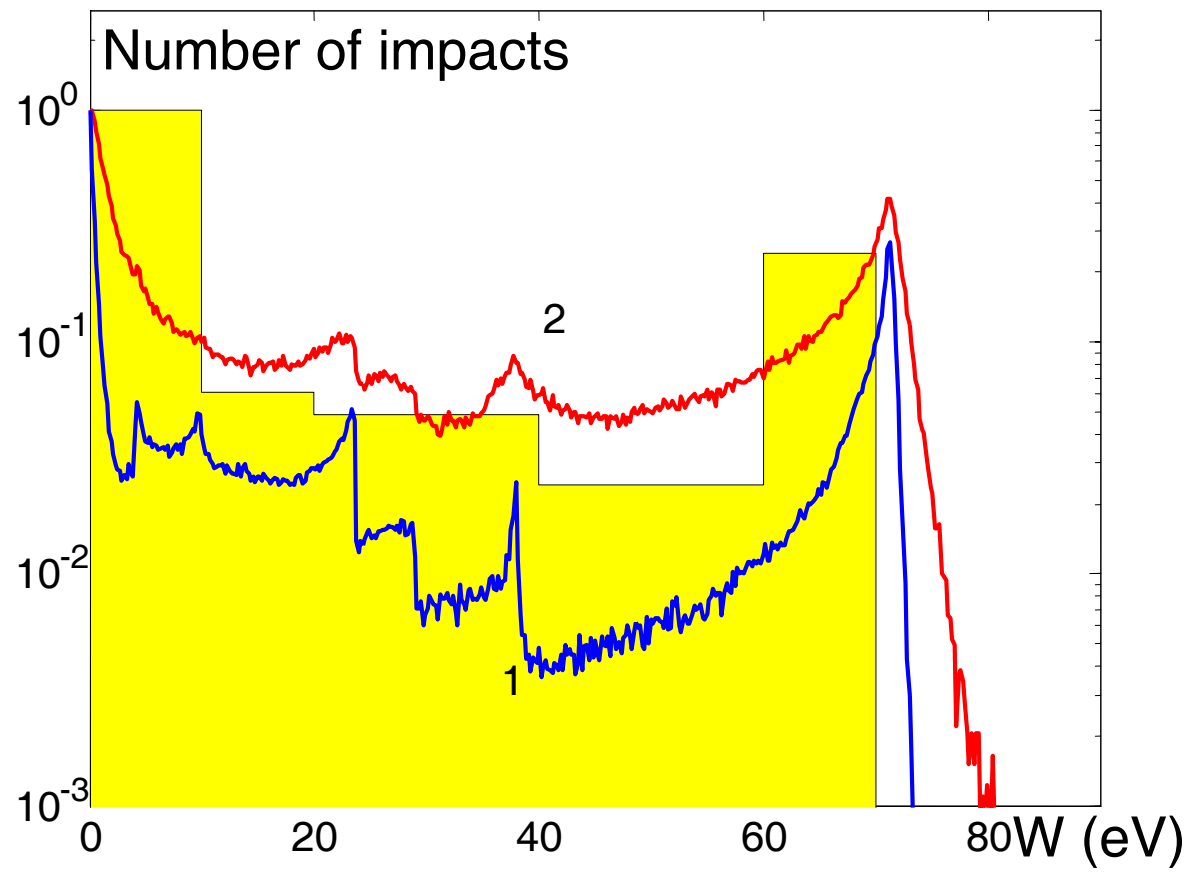

FIG. 12: The distribution of electron impact energy at the outer electrode (normalized to the maximum value). The presented results are averaged over the last 8 (i.e. from 92 up to 100) microwave periods. The simulations are carried out using the same parameters as in Figs. 10-11. Line 1 corresponds to $\sigma_{\max }=2, W_{1}=11 \mathrm{eV}$ and $W_{s}=1 \mathrm{eV}$ and line 2 to $\sigma_{\max }=2$, $W_{1}=20 \mathrm{eV}$ and $\left.W_{s}=5 \mathrm{eV}\right)$. Superimposed are the experimental results presented in Fig.6

walls oscillates with a phase shift of $\pi / 2$ with respect to the microwave field oscillations.

At first sight a serious argument against the poly-phase nature of the multipactor seems to be the fact that the numerical simulations indicate a considerable influence of the electron initial energy on the multipactor threshold. When applied to the planeparallel model, the poly-phase theory [27] does not predict such an effect since the contribution of the electron initial energy to the impact energy is relatively weak. However, the situation changes drastically when the multipactor occurs in a coaxial waveguide. As found in previous studies [23, 35, 36], depending on parameters, two qualitatively different regimes of multipactor (two-sided and one-sided) are possible in a coaxial waveguide. Specifically, when the initial electron velocity is neglected and the ratio of outer to inner radii exceeds $\sqrt{3}$, theory predicts that only one-sided multipactor (localized at the outer electrode) is possible [35]. This result is caused by the action 
of the Miller (or ponderomotive) force which tends to push electrons out of regions with strong rf field (here the vicinity of the inner electrode). Clearly, a finite initial velocity makes it possible for an electron to overcome the Miller repulsion and shifts the border between the two-sided and one-sided multipactor regimes towards higher values of $R_{\text {out }} / R_{\text {in }}$. In our experiments, this ratio was taken as $R_{\text {out }} / R_{\text {in }}=2$ and consequently an increase in electron energy from $1 \mathrm{eV}$ up to $5 \mathrm{eV}$ corresponds to a transition from a multipactor which is mainly one-sided to one that is mainly two-sided. This conclusion is confirmed by numerical simulations (cf. Fig. 11) and makes it possible to understand why the multipactor threshold is so sensitive to electron initial energy.

In order to understand the observed distribution of electron impact velocities, we emphasize the following points:

(i) A spread of electron initial velocity results in a considerable spread of the corresponding flight times and therefore an important part of the electron impacts at "'wrong"' phases of the microwave field i.e. at moments when this field does not accelerate secondary electrons out from the surface of emission, but instead pushes them back to this surface. Such secondary electrons return back in a short time with an energy close to their initial energy and contribute to the peak of low energy electron impacts (Fig. 12). The low energy peak contains approximately half of all impacts whereas its width is about the average initial energy of the secondary electrons.

(ii) When a secondary electron starts from the outer electrode and returns back to this electrode, its maximum impact energy is $2 m V_{\omega}^{2}$ [35], where $V_{\omega} e E / m \omega$ is the electron oscillatory velocity at the outer electrode $(e$ and $m$ denote electron charge and mass respectively, $\omega$ is the circular field frequency and $E$ denotes the electric field amplitude at the outer electrode). At the input power, $P=1.5 \mathrm{~kW}$, the maximum impact energy of returning electrons is estimated to be $2 m V_{\omega}^{2} \approx 24 \mathrm{eV}$, which corresponds to one of the intermediate peaks in Fig. 12.

(iii) When a secondary electron starts from the inner electrode and then collides with the outer electrode, its maximum impact energy can also be estimated using the approximate theory of electron motion in a coaxial waveguide [35] with the result $m V_{\omega}^{2}(1+\sqrt{5.5})^{2} / 2 \approx 67 \mathrm{eV}$, which corresponds to the peak with high impact energy in Fig. 12 . 
The above simple analysis implies the conclusion that the contribution to the multipactor avalanche from electrons which transit between the electrodes is important even in the considered case of small initial energy of the electrons.

\section{CONCLUSION}

An experimental study and numerical simulations have been carried out of the multipactor discharge in a coaxial waveguide in vacuum and the obtained results have been analyzed and compared. This comparison makes it possible to determine the parameters of the secondary emission yield of the waveguide walls in the experiment. It is also possible to conclude that the multipactor discharge observed in the experiment is excited in the poly-phase regime and that both one-sided and two-sided multipactor avalanches contribute to the discharge.

\section{ACKNOWLEDGMENT}

This work was supported in part by the Russian Foundation for Basic Research through grant number 09-02-97024

[1] Y. Ikeda, T. Imai and K. Sakamoto, Discharge at the pillbox window for an LHRF launcher, IEEE Trans. Plasma Science 17 (3), 534-540 (1989).

[2] A. Woode and J. Petit, Investigations into multipactor breakdown in satellite microwave payloads, ESA Journal 14 (1), 467-478 (1990).

[3] N. Rozario, H. F. Lenzing, K. F. Reardon, M. S. Zarro C. G. Baran, Investigation of Telstar 4 spacecraft Ku-band and C-band antenna components for multipactor breakdown, IEEE Trans. Microwave Theory and Technique 42 (4), 558-564 (1994).

[4] N. F. Kovalev, V. E. Nechaev, M. I. Petelin, N. I. Zaitsev, Scenario for output pulse shortening in microwave generators driven by relativistic electron beams, IEEE Trans. Plasma Science 36(3), 246-251 (1998). 
[5] A. Neuber, J. Dickens, D. Hemmert, H. Krompholz, L. L. Hatfield, and M. Kristiansen, Window breakdown caused by high-power microwaves, IEEE Trans. Plasma Science 26 (3), 296-303 (1998).

[6] J. G. Power, W. Gai, S. H. Gold, A. K. Kinkead, R. Konecny, C. Jing, W. Liu, and Z. Yusof, Observation of multipactor in an alumina-based dielectric-loaded accelerating structure, Phys. Rev. Letters 92(16), 164801 (1-4) (2004).

[7] T. Abe, T. Kageyama, K. Akai, K. Ebihara, H. Sakai, and Y. Takeuchi, Multipactoring zone map of an rf input coupler and its application to high beam current storage rings, Physical Review Special Topics - Accelerators And Beams 9, 062002 (1-8) (2006).

[8] J. R. M. Vaughan, Multipactor, IEEE Trans. Electron Devices 35(7), 1172-1180 (1988).

[9] R. A. Kishek, Y. Y. Lau, L. K. Ang, A. Valfells, and R. M. Gilgenbach, Multipactor discharge on metal and dielectrics: Historical review and recent theories, Phys. Plasmas $5(5), 2120-2126$ (1998).

[10] S. K. Nagesh, D. Revannasiddiah and S. V. K. Shastry, Investigation of multipactor breakdown in communication satellite microwave co-axial systems, PRAMANA - Journal of Physics 64(1), 95-110 (2005).

[11] J. de Lara, F. Perez, M. Alfonseca, L. Galan, I. Montero, E. Roman and D. Raboso. Multipactor prediction for on-board spacecraft RF equipment with the MEST software tool, IEEE Trans. Plasma Science 34(2), 476-484 (2006).

[12] M. Perez, C. Tienda, C. Vicente, A. Coves, G. Torregrosa, B. Gimeno, R. Barcot, V. E. Boria and D. Raboso, Multipactor analysis in coaxial waveguides for satellite applications using frequency-domain methods, 2006 IEEE MTT-S International Microwave Symposium Digest (IEEE Cat. No. 06CH37734C), 1045-1048 (2006) .

[13] M. Yu, Power-handling capability for rf filters, IEEE Microwave Magazine 8(5), 88-97 (2007).

[14] M. A. Gusarova, V. I. Kaminsky, L.V. Kravchuk, S. V. Kutsaev, M. V. Lalayan, N. P. Sobenin and S. G. Tarasov, Multipacting simulation in accelerating RF structures, Nuclear Instruments and Methods in Physics Research A 599, 100-105 (2009).

[15] O. V. Sinitsyn, G. S. Nusinovich, and T. M. Antonsen, Jr., Self-consistent nonstationary two-dimensional model of multipactor in dielectric-loaded accelerator structures, Physics 
of Plasmas 16(7), 073102 (1-9) (2009).

[16] A. M. Perez, V. E. Boria, B. Gimeno, S. Anza, C. Vicente, J. Gil, Multipactor analysis in circular waveguides, J. Electromagnetic Waves and Applications 23(11-12), 1575-1583 (2009).

[17] V. E. Semenov, E. I. Rakova, A. G. Sazontov, I. M. Nefedov, V. I. Pozdnyakova, I. A. Shereshevskii, D. Anderson, M. Lisak and J. Puech, Simulations of multipactor thresholds in shielded microstrip lines, J. Phys. D: Appl. Phys. 42(20), 205204 (1-7) (2009).

[18] A. M. Perez, C. Tienda, C. Vicente, S. Anza, J. Gil, B. Gimeno, V. E. Boria, and D. Raboso, Prediction of multipactor breakdown thresholds in coaxial transmission lines for travelling, standing and mixed waves, IEEE Trans. Plasma Science 37(10), 2031-2040 (2009).

[19] ESA for ECSS, Space engineering, multipactor design, and test, ESA Publications Division, Noordwijk, The Netherlands, ECSS-E-20-01-01A (2003).

[20] I. A. Kossyi, G. S. Lukyanchikov, V. E. Semenov, E. I. Rakova, D. Anderson, M. Lisak and J. Puech, Polyphase (non-resonant) multipactor in rectangular waveguides, J. Phys. D: Applied Phys. 41(6), 065203 (1-8) (2008).

[21] R. Woo and A. Ishimaru, A similarity principle for multipacting discharges, J. Applied Physics 38(13), 5240-5244 (1967).

[22] R. Woo, Multipacting discharges between coaxial electrodes, J. Applied Physics 39(3), 1528-1533 (1968).

[23] E. Somersalo, P. Yla-Oijala and D. Proch, Analysis of multipacting in coaxial lines, Proc. 1995 Particle Accelerator Conference (Cat. No.95CH35843) 3, 1500-1502 (1995).

[24] T. P. Graves, B. LaBombard, S. Wukitch and I. Hutchinson, The coaxial multipactor experiment (CMX): A facility for investigating multipactor discharges, Rev. Scientific Instruments 77, 014701(1-4) (2006).

[25] G. S. Luk'yanchikov, Multiphase uniform secondary-emission microwave discharge at a solid surface, Sov. Phys. Tech. Phys. 19(9), 1196-1199 (1975).

[26] A. A. Dorofeyuk, I. A. Kossyi, G. S. Luk'yanchikov and M. M.Savchenko, Study of the electron discharge caused by the interaction of microwave radiation with metal surfaces, Sov. Phys. Techn. Phys. 21(1), 76-80 (1976). 
[27] L. V. Grishin, A. A. Dorofeyuk, I. A. Kossyi, G. S. and M. M. Savchenko, A study of secondary-emission microwave discharges with large electron transit angles, Lebedev Physics Institute Series (Consultants Bureau, New York, London, 1977) 92, 63-101 (1977).

[28] L. V. Grishin and G. S. Luk'yanchikov, Multipactor discharge with an electron velocity distribution, Sov. Phys. Tech. Phys. 21(3), 307-311 (1976).

[29] A. Sazontov, M. Buyanova, V. Semenov, E. Rakova, V. Vdovicheva, D. Anderson, M. Lisak, J. Puech and L. Lapierre, Effect of emission velocity spread of secondary electrons in two-sided multipactor, Phys. Plasmas 12(5), 053102 (1-8) (2005).

[30] G. M. Batanov, E. F. Bol'shakov, A. A. Dorofeyuk, I. A. Kossyi, A. V. Sapozhnikov, V. A.Silin, V. G. Brovkin, Yu. F. Kolesnichenko, A. N. Voronin, N. Koumvakalis, S. Holly, T. L. Bunn, Plasma formation during solid-body irradiation by microwaves and its application for localizing the energy input, J. Phys. D: Appl. Phys. 29(6), 1641-48 (1996).

[31] G. M. Batanov, N. K. Berezhetskaya, I. A. Kossyi, A. N. Magunov and V. P. Silakov, Interaction of high-power microwave beams with metal-dielectric media, Eur. Phys. J. Applied Physics, 26(1), 11-16 (2004).

[32] A. Kryazhev, M. Buyanova, V. Semenov, D. Anderson, M. Lisak, J. Puech, L. Lapierre and J. Sombrin, Hybrid resonant modes of two-sided multipactor and transition to the polyphase regime, Phys. Plasmas 9(11), 4736-4743 (2002).

[33] R. Udiljak, D. Anderson, M. Lisak, V. E. Semenov and J. Puech, Multipactor in a coaxial transmission line. II. Particle-in-cell simulations, Phys. Plasmas 14(3), 033509 (1-7) (2007).

[34] J. R. M. Vaughan, A new formula for secondary emission yield, IEEE Trans. Electron Devices 36(9), 1963-1967, (1989).

[35] R. Udiljak, D. Anderson, M. Lisak, V. E. Semenov and J. Puech, Multipactor in a coaxial transmission line. I. Analytical study, Phys. Plasmas 14(3), 033508 (1-11) (2007).

[36] K. Sakamoto, Y. Ikeda, and T. Imai, Numerical study of RF discharge caused by secondary electron emission, J. Phys. D: Appl. Phys. 22(12), 1840-1847 (1989). 